\title{
Dificuldades de acesso ao tratamento de pacientes com indicação de cirurgia de catarata nos Sistemas de Saúde Público e Privado
}

\author{
Difficulties in access to treatment for patients undergoing cataract surgery \\ in public and private health systems
}

Newton Kara-Júnior ${ }^{1}$, Roberto Dellapi JR. ${ }^{1}$, Rodrigo França de Espíndola ${ }^{1}$

\section{RESUMO}

Objetivo: Estimar o valor dos Projetos Catarata para a comunidade, identificando características e dificuldades de acesso ao diagnóstico e ao tratamento da catarata na rotina de atendimento de diversos Sistemas de Saúde.

Métodos: Durante uma campanha de catarata realizada em um hospital universitário foi aplicado um questionário de múltipla escolha somente aos pacientes selecionados para a cirurgia de catarata. Foram avaliadas, dentre outras, as seguintes variáveis: acesso prévio à consulta oftalmológica (serviço público ou privado); motivo(s) para a não realização da cirurgia no serviço inicial.

Resultados: Foram avaliados 627 pacientes com diagnóstico de catarata. A maioria 595 (95\%) já havia consultado um oftalmologista previamente, sendo que em 63\% das situações (375 pacientes) a consulta havia sido realizada há menos de um ano. A última avaliação oftalmológica foi realizada pelo Sistema Único de Saúde (SUS) em 52\% dos casos (307 pacientes), e entre estes, a fila de espera foi apontada pela maioria como sendo a causa da não realização da cirurgia. Com relação aos pacientes previamente atendidos em serviços privados, o motivo da não realização da cirurgia foi o custo da cirurgia e custo da lente intraocular.

Conclusão: Os resultados deste estudo sugerem que a rotina de atendimento oftalmológico no SUS em São Paulo não está preparada para atender a demanda por cirurgias de catarata, e o sistema de saúde privado ainda exclui uma parcela da população que possuí acesso à consulta clínica da cirurgia de catarata. É importante a continuidade da realização de campanhas comunitárias para atender a população que não teria como acessar a cirurgia pelas vias convencionais.

Descritores: Catarata; Extração de catarata; Cegueira/prevenção \& controle; Acesso aos serviços de saúde; Custos de cuidados de saúde/estatística e dados numéricos; Questionários; Brasil

\begin{abstract}
Purpose: To identify the difficulties in access to diagnosis and treatment of cataract in patients attended during a cataract campaign

Methods: A questionnaire was administered to patients selected for cataract surgery. We evaluated, among others, the following variables: prior access to ophthalmologists (public or private), reason(s) for not having surgery early in the initial service, the average cost charged by cataract surgery and intraocular lens (IOL) (private services and agreements).

Results: A total of 627 patients was evaluated. Most of them - 595 (95\%) had previously attended an ophthalmologist, and in 63\% of cases (375 patients) the consultation had been held for less than a year. The last evaluation was performed by the Brazilian Public Health System (SUS) in 52\% of the cases (307 patients). Regarding the reason for not having surgery in the initial service, the high cost of the surgery $(R \$ 2.000-R \$ 4.000)$ and the cost of IOLS (R\$1.000-R\$1.500) was the main obstacle for most attended services and private covenants.

Conclusion: The results of this study suggest that the routine of ophthalmologic care in SUS at São Paulo is not prepared to answer the demand for cataract surgeries and private healthcare system still excludes a portion of the population. It is important to continue the implementation of community campaigns to serve the population that would be unable to access the surgery through conventional ways.
\end{abstract}

Keywords: Cataract; Cataract extration; Blindness/prevention \& control; Health services accessibility; Health care costs/statistics \& numerical data; Questionnaires; Brazil

\section{INTRODUÇÃO}

Estima-se que a catarata seja responsável por $50 \%$ dos casos de cegueira no mundo(1-6). A perda da capacidade visual além de comprometer a qualidade de vida dos pacientes, acarreta prejuízo para a sociedade ${ }^{(7-8)}$

A cirurgia da catarata apresenta alta eficiência, favorável custobenefício no tratamento da doença e na reabilitação visual do indivíduo e oferece grande impacto para a sociedade ${ }^{(9-11)}$.

Nos países em desenvolvimento, mesmo nas grandes capitais, o acesso de pacientes necessitados à cirurgia de catarata é dificultado devido à fatores socioeconômicos e culturais, além de obstácu- los criados pelo próprio sistema de saúde, o que impede a realização de mais procedimentos cirúrgicos ${ }^{(10-15)}$. Até mesmo em países desenvolvidos existem barreiras para a realização da facectomia, a falta de recursos financeiros e de seguros médicos são apontados como significantes obstáculos ${ }^{(16)}$.

Em 1998 foi instituída a Campanha Nacional de Catarata. O acesso ao tratamento foi facilitado com a realização de ProjetosCatarata em todo o país e o Governo Federal determinou que o financiamento das cirurgias pelo SUS fosse em forma de "extrateto" para as instituições credenciadas, garantindo o financiamento de todas as cirurgias realizadas. O Projeto Catarata procura eliminar
Funding: No specific financial support was available for this study.

Disclosure of potential conflicts of interest: N.Kara-Júnior, None; R.Dellapi Jr., None; R.F.de Espíndola, None.

Correspondence address: Rodrigo França Espíndola. Praça das Hortências, 70 - Condomínio Portal de Itu - Itu - (SP) - 13301-689 - Brazil - E-mail: rodrigo166@uol.com.br 
obstáculos logísticos para que o indivíduo deficiente visual por catarata receba o tratamento necessário mediante a facilitação do acesso ao exame oftalmológico e à cirurgia, assim como através de processo educativo da população. Contudo, em 2006 o Governo Federal alegando falta de verba, descontinuou o repasse "extra-teto" e desestimulou a realização dos Projetos Catarata $(4,10,11,15)$.

O objetivo desse estudo é estimar o valor dos Projetos Catarata para a comunidade, identificando características e dificuldades de acesso ao diagnóstico e ao tratamento da catarata na rotina de atendimento de diversos Sistemas de Saúde, em pacientes que somente acessaram a cirurgia por meio de Projetos Catarata.

\section{MÉTODOS}

Realizou-se um estudo transversal somente entre pacientes com diagnóstico de catarata realizado durante um Projeto Catarata realizado em agosto de 2005 no Hospital das Clínicas da Universidade de São Paulo (HC-FMUSP). A pesquisa foi baseada na coleta de dados de um questionário de múltipla escolha padronizado fornecido somente a pacientes selecionados para a cirurgia.

As seguintes variáveis foram analisadas: sexo; idade; grau de escolaridade; acesso à consulta oftalmológica prévia (serviço público ou privado); motivo(s) para a não realização da cirurgia no serviço inicial, e para a procura da campanha de catarata. Outros dados ainda foram coletados: gastos com alimentação e transporte durante a campanha; custo médio da cirurgia de catarata (para atendidos em serviços particulares); custo médio da lente intraocular (para atendidos em convênios/seguros de saúde).

A amostragem foi por conveniência (não probabilística), e o número de pacientes analisados foi estabelecido arbitrariamente. A análise estatística baseou-se em dados descritivos da amostra, sendo as variáveis descritas por meio de médias ou proporções.

Os questionários foram aplicados pelos próprios autores que não tiveram influência nas respostas. Os entrevistados foram orientados a não mudarem ou rasurarem suas respostas. Questões deixadas em branco ou rasuradas foram desconsideradas.

O consentimento de todos os entrevistados foi obtido para a publicação dos dados desse estudo.

\section{RESULTADOS}

Foram avaliados 627 pacientes com diagnóstico de catarata, destes 373 (59\%) eram do sexo feminino. A idade variou de 40 a 96 anos, com média de 67,8 $\pm 8,2$ anos. Quanto à escolaridade, grande parte dos entrevistados 348 (55\%) possuía até o ensino fundamental (Tabela 1).

A maioria 595 (95\%) já havia consultado um oftalmologista antes, sendo que em $63 \%$ dos casos (375 pacientes) a consulta havia sido realizada há menos de um ano.

Com relação à última avaliação oftalmológica, aproximadamente metade dos casos (52\%) foi realizada pelo Sistema Único de Saúde (SUS) (Tabela 2). Na ocasião desta consulta o diagnóstico de catarata com indicação cirúrgica havia sido feito em 86\% dos casos (514 pacientes)

Em relação ao motivo da não realização da cirurgia no serviço de origem, dentre aqueles que procuraram o SUS, a maioria não a fez devido à fila de espera; dos que procuraram o atendimento particular, a não realização se deu por causa do custo da cirurgia; e dentre aqueles que foram avaliados pelo sistema de saúde suplementar, o custo da lente intraocular (LIO) foi o maior obstáculo (Tabela 3).

No caso dos pacientes que procuraram inicialmente um serviço privado (202 indivíduos), somente uma parcela foi apta a responder sobre o custo da cirurgia de catarata (56 indivíduos). A maioria desses (51,7\%), informou que o custo situava-se entre $R \$ 2.000$ e $\mathrm{R} \$ 4.000,19$ pacientes $(34,0 \%)$ informaram que o custo era menos de $R \$ 2.000$ e 8 indivíduos (14,3\%) afirmaram que o custo era superior a $R \$ 4.000$

Dentre os pacientes que possuíam convênio/seguros de saúde (82 indivíduos), a maioria informou que o custo da LIO foi determinante para a não realização da cirurgia (Tabela 3). Dentre estes, somente uma parcela foi apta a responder sobre o custo do insumo cirúrgico (34 indivíduos). A maioria desses (53,0\%), informou que o custo situava-se entre $\mathrm{R} \$ 1.000$ e $\mathrm{R} \$ 1.500,8$ pacientes $(23,5 \%)$ informaram que o custo situava-se entre $\mathrm{R} \$ 500$ e $\mathrm{R} \$ 1.000$ e 8 indivíduos $(23,5 \%)$ informaram ser o custo superior a $\mathrm{R} \$ 1.500$.

A tabela 4 demonstra o gasto médio dos pacientes para participar da campanha de catarata (alimentação/transporte).

Tabela 1. Características dos pacientes atendidos durante a campanha de catarata no HC-FMUSP $(n=627)$

\begin{tabular}{lcc}
\hline Características & $\mathbf{n}$ & \% \\
\hline Sexo & & \\
Masculino & 254 & 40,5 \\
Feminino & 373 & 59,5 \\
Escolaridade & & \\
Analfabeto & 169 & 26,9 \\
Fundamental & 348 & 55,5 \\
Ensino médio & 72 & 11,5 \\
Ensino superior & 38 & 6,1 \\
\hline
\end{tabular}

Tabela 2. Tipo de serviço procurado por pacientes que já haviam sido consultados por um oftalmologista $(n=595)$

\begin{tabular}{lcc}
\hline Serviço & n & \% \\
\hline SUS & \multicolumn{1}{c}{} & 51,6 \\
Particular & 202 & 34,0 \\
Convênios/seguro de saúde & 86 & 14,4 \\
\hline
\end{tabular}

SUS = Sistema Único de Saúde

Tabela 3. Motivo da não realização da cirurgia da catarata no serviço em que foi feito o diagnóstico inicial (particular, convênios, SUS)

\begin{tabular}{|c|c|c|c|}
\hline & Particular $(n=202)$ & Convênios $(n=82)$ & SUS $(n=241)$ \\
\hline Motivo & $\%$ & $\%$ & $\%$ \\
\hline Falta de recursos financeiros & 63,2 & 63,4 & NA \\
\hline Fila de espera & 3,9 & 4,8 & 30,3 \\
\hline Não conseguiu marcar a cirurgia & 2,4 & 3,6 & 19,5 \\
\hline Medo & 6,8 & 6,1 & 11,6 \\
\hline Falta de confiança no médico & 3,9 & 3,6 & 4,1 \\
\hline Catarata incipiente & 12,2 & 10,9 & 19,5 \\
\hline Outros & 7,3 & 7,3 & 14,9 \\
\hline
\end{tabular}

SUS= Sistema Único de Saúde; NA= não se aplica; O valor de "n" não corresponde ao número total de pacientes em cada grupo, pois alguns assinalaram respostas múltiplas 
Tabela 4. Gasto médio (transporte/alimentação) dos pacientes selecionados para a cirurgia de catarata para participar da campanha no HC-FMUSP $(n=627)$

\begin{tabular}{lcc}
\hline Gastos & $\mathbf{n}$ & \% \\
\hline Sem gastos & 262 & 41,8 \\
Até R\$ 10 & 215 & 34,3 \\
Entre R\$ 10 e R\$ 20 & 78 & 12,4 \\
Mais de R\$ 20 & 72 & 11,5 \\
\hline
\end{tabular}

\section{DISCUSSÃO}

A intervenção pública de saúde na forma de projeto comunitário para a reabilitação da visão produz resultados que, além de prevenir a cegueira, tem impacto sobre a qualidade de vida dos indivíduos, com importantes benefícios sociais ${ }^{(8)}$

Estimativas apontam que o Brasil não consegue realizar o número de cirurgias de catarata necessárias para compensar o surgimento de novos casos, com isso cresce a importância dos Projetos Cataratas e da otimização dos centros cirúrgicos ambulatoriais para as cirurgias em larga escala ${ }^{(15,17)}$.

Assim como em outros estudos, a maioria dos pacientes atendidos nesta campanha apresentava baixa escolaridade e já havia sido examinado em outro serviço oftalmológico(18-24), sugerindo haver facilidade de acesso à consulta oftalmológica, porém dificuldade em se acessar a cirurgia corretiva. Esta pesquisa estima que, principalmente para população dependente do SUS, as campanhas comunitárias realizadas para triagem e tratamento da catarata são importantes para compensar alguns obstáculos logísticos de acesso à cirurgia, considerando-se que 52\% destes pacientes com indicação cirúrgica já haviam procurado atendimento público, e não haviam sido operados.

Os principais motivos para a não realização da cirurgia de catarata no atendimento inicial foram a falta de recursos financeiros (serviços particulares e convênios) e para aqueles atendidos pelo SUS, a fila de espera foi apontada como a principal causa. É importante notar, que embora a maioria já possuía o diagnóstico de catarata, muitos esperavam o chamado para a cirurgia. As campanhas possuem um papel crucial no combate a cegueira, pois em um mesmo dia os pacientes são diagnosticados e têm o dia da cirurgia agendada, diminuindo retornos desnecessários ao hospital e minimizando o tempo para o tratamento.

Outro estudo realizado na região nordeste do Brasil, também constatou que metade dos pacientes já haviam procurado o sistema público de sáude e que $40 \%$ dos atendidos foram a um serviço privado, porém ainda não haviam sido operados ${ }^{(25)}$. Nota-se portanto, que tando na região sudeste como na nordeste, apesar de possuirem realidades diferentes, o acesso à consulta oftalmológica não se mostrou um problema, mesmo assim os pacientes continuavam com baixa visão devido a catarata, demonstrando uma baixa resolutividade, tanto do sistema público como do privado.

Para comparecer ao Projeto Catarata, a maioria dos indivíduos $(76,1 \%)$ teve despesas de até $\mathrm{R} \$ 10$, relativa a gastos com transporte e alimentação. Como no dia da campanha são realizados todos os exames necessários para a cirurgia, os pacientes não precisam retornar ao hospital até a data marcada para o procedimento, reduzindo-se, assim, algumas barreiras para a realização da cirurgia (falta às consultas, gastos extras, disponibilidade de acompanhante, etc.).

Com relação aos indivíduos que possuíam condições de atendimento pelo Setor Privado de Saúde, o Projeto Catarata foi importante para suprir as necessidades daqueles que não puderam arcar com os custos da cirurgia. Alguns indivíduos que possuíam convênio/seguro saúde, também procuraram a campanha comunitária devido ao custo do insumo cirúrgico (LIO), que para a maioria dos entrevistados situou-se entre $R \$ 1.500$ a $R \$ 2.000$.

\section{CONCLUSÃO}

Os resultados deste estudo sugerem que a rotina de atendimento oftalmológico no Sistema Público de Saúde, em São Paulo, não está preparada para atender a demanda por cirurgias de catarata. E que o Sistema de Saúde Privado ainda exclui uma parcela da população que tem acesso à consulta clínica, da cirurgia de catarata. Enfatizando a necessidade de continuar a realização de campanhas comunitárias para atender a população que não teria como acessar a cirurgia pelas vias convencionais.

\section{REFERÊNCIAS}

1. McCarty CA, Keeffe JE, Taylor HR. The need for cataract surgery: projections based on lens opacity, visual acuity, and personal concern. Br J Ophthalmol. 1999;83(1):62-5.

2. Resnikoff S, Pascolini D, Etya'ale D, Kocur I, Pararajasegaram R, Pokharel GP. Global data on visual impairment in the year 2002. Bull World Health Organ. 2004;82(11):844-51.

3. Javitt JC, Wang F, West SK. Blindness due to cataract: epidemiology and prevention. Ann Rev Pub Health. 1996;17:159-77

4. Arieta CE, Kara-José N, Alves MR. Estratégias para a ação contra a catarata. In: Kara-José N, Delgado AMN, Arieta CEL, Rodrigues MLV, Alves MR, editores. Prevenção da cegueira por catarata. Campinas: UNICAMP, 1996. p.19-35.

5. Ellwein LB, Kupfer C. Strategic issues in preventing cataract blindness in developing countries. Bull World Health Organ. 1995;73(5):681-90.

6. Thylefors B, Négrel AD, Pararajasegaram R, Dadzie KY. Global data on blindness. Bull World Health Organ. 1995;73(1):115-21.

7. Kara-Junior N, Mazurek MG, Santhiago MR, Parede TR, Espindola RF, Carvalho RS. Phacoemulsification versus extracapsular extraction: governmental costs. Clinics. 2010; 65 (4):357-61.

8. Kara-Junior N, Parede TRR, Santiago MR, Espindola RF, Mazurek MGG, Carvalho RS. Custo social de duas técnicas de cirurgia de catarata no Brasil. Rev Saúde Pública. 2010; 44(5):957-62.

9. Batterbury M, Khaw PT, Hands R, Elkington AR. The cataract explosion: the changing pattern of diagnoses of patients attending an ophthalmic outpatient department. Eye (Lond). 1991;5(Pt 3):369-72.

10. Kara-José N, Temporini ER. [Cataract surgery: or why are there some patients excluded]. Rev Panam Salud Publica. 1999;6(4):242-8. Portuguese.

11. Kara-José N, Arieta CEL, Temporini ER. Tratamento cirúrgico de catarata senil: óbices para o paciente. Arq Bras Oftalmol. 1996;59(6):573-7.

12. Schwab L. Public health, preventive care, and eye care delivery and evaluation. In: Schwab $L$, editor. Eye care in developing nations. $3^{\text {rd }}$ ed. San Francisco: The Foundation of the American Academy of Ophthalmology, 1999. p. 3-17.

13. Rehder JR, Ribeiro LG, Melo Filho PA, Aoki RH. "Projeto Catarata": uma solução para saúde pública ocular. Rev Bras Oftalmol. 1990;58(4):271-5.

14. Temporini ER, Kara-José N, Kara-José Jr N. Catarata senil: características e percepções de pacientes atendidos em projeto comunitário de reabilitação visual. Arq Bras Oftalmol. 1997;60(1):79-83.

15. Kara-Junior N, Espíndola RF. Evolução e viabilização de um centro cirúrgico ambulatorial para cirurgias de catarata em larga escala em um hospital universitário. Arq Bras Oftalmol. 2010; 73(6):494-6.

16. Broman AT, Hafiz G, Muñoz B, Rodriguez J, Snyder R, Klein R, et al. Cataract and barriers to cataract surgery in a US Hispanic population: Proyecto VER. Arch Ophthalmol. 2005; 123(9):1231-6.

17. Kara-Junior N, Santhiago MR, Espíndola RF. Importância do centro cirúrgico ambulatorial para a realização de cirurgias de catarata em larga escala. Rev Bras Oftalmol. 2011; 70(2):75-6.

18. Ferraz EVA, Lima CA, Cella W, Arieta CEL. Adaptação de questionário de avaliação da qualidade de vida para aplicação em portadores de catarata. Arq Bras Oftalmol. 2002; 65(3):293-8.

19. Pokharel GP, Selvaraj S, Ellwein LB. Visual functioning and quality of life outcomes among cataract operated and unoperated blind populations in Nepal. Br J Ophthalmol. 1998;82(6):606-10.

20. Rezende Filho F, Rezende F. Histórico. In: Rezende F. Cirurgia de catarata. Rio de Janeiro: Cultura Médica; 2001. p.3-8.

21. Leite Arieta CE, José NK, Carvalho Filho DM, Ruiz Alves M. Optimization of a university cataract-patient care service in Campinas, Brazil. Ophthalmic Epidemiol. 1999;6(2):113-23.

22. Kara-José Jr. N, Schellini SA, Silva MR, Bruni LF, Almeida AG. Projeto Catarata: qual a sua importância para a comunidade? Arq Bras Oftalmol. 1996;59(5):490-3.

23. Malerbi FK, Lopes JF, Ajimura FY, Alves MR, Temporini ER. Barreiras ao acesso no tratamento da catarata senil: aspectos sócio-culturais. Rev Bras Oftalmol. 2000;59(9):649-54.

24. Lima DMG, Ventura LO, Brandt CT. Barreiras para o acesso ao tratamento da catarata senil na Fundação Altino Ventura. Arq Bras Oftalmol 2005;68(3):357-62.

25. Lima DMG, Ventura LO, Brandt CT. Barreiras para o acesso ao tratamento da catarata senil na Fundação Altino Ventura. Arq Bras Oftalmol. 2005;68(3):357-62. 